man in der Mehrzahl der Falle mit einer sehr kleinen $\mathrm{Zahl}$ derartiger Verbindungen auskommt.

Folgendermaßen kann man wenigstens die GesetzmäBigkeiten erster Ordnung in einem Bilde vereinigen. Man denkt sich die Zusammensetzung umgerechnet auf Molekularprozente von $\left(\mathrm{SiO}_{2}+\right.$ $\left.\mathrm{TiO}_{2}\right), \quad\left(\mathrm{Na}_{2} \mathrm{O}+\mathrm{K}_{2} \mathrm{O}\right), \quad(\mathrm{FeO}+\mathrm{MgO}+\mathrm{CaO})$, $\mathrm{Al}_{2} \mathrm{O}_{3}{ }^{1}$ ). In dem von diesen vier Stoffgruppen gebildeten Konzentrationstetraeder nehmen die typischen Eruptivgesteine nur einen kleinen Raum ein. Er erstreckt sich vom $\mathrm{SiO}_{2}-\mathrm{Pol}$ aus gegen die Verbindungen $\left[2(\mathrm{RIO}) . \mathrm{SiO}_{2}\right] ;\left[\mathrm{RIAl}_{2} \mathrm{O}_{4} .2 \mathrm{SiO}_{2}\right]$; $\left[\mathrm{RI}_{2} \mathrm{Al}_{2} \mathrm{O}_{4} \cdot 2 \mathrm{SiO}_{2}\right]$. Hauptsächlich infolge der $\mathrm{Ver}$ rechnung. von $\mathrm{Fe}_{2} \mathrm{O}_{3} \mathrm{zu} \mathrm{FeO}$ statt $\mathrm{zu} \mathrm{Al}_{2} \mathrm{O}_{3}$ ist es notwendig, den Raum gegen den Pol der Alkalien

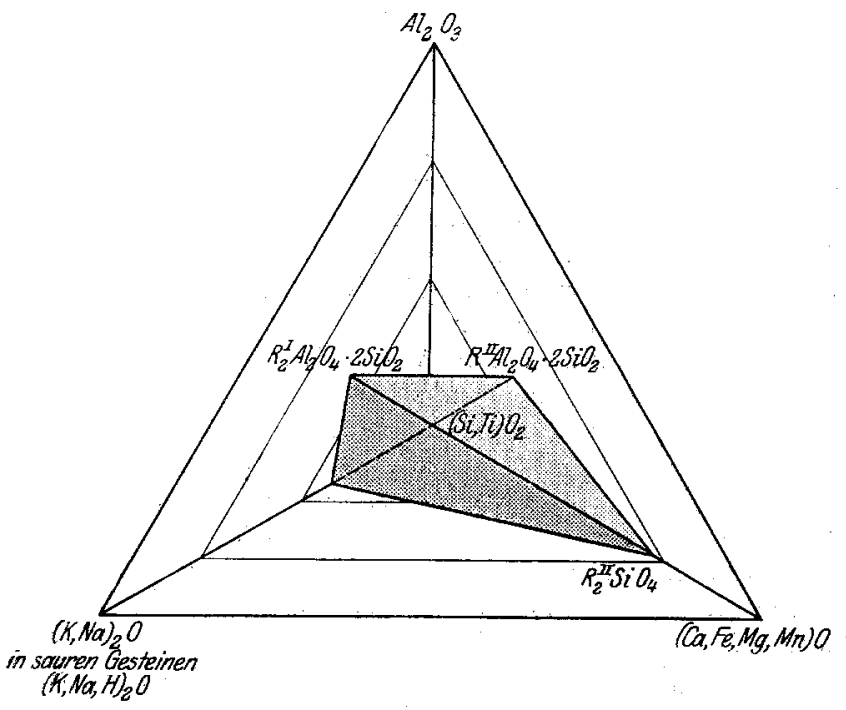

Fig. 5. Raum für typische Eruptivgesteine im Konzentrationstetraeder

$\mathrm{Al}_{2} \mathrm{O}_{3}:(\mathrm{K}, \mathrm{Na})_{2} \mathrm{O}:(\mathrm{Ca}, \mathrm{Fe}, \mathrm{Mg}, \mathrm{Mn}) \mathrm{O}:(\mathrm{Si}, \mathrm{Ti}) \mathrm{O}_{2}$.

hin zu erweitern, entsprechend dem Auftreten ägirinartiger Moleküle. Die Abgrenzung kann nach dieser Seite ohne umfassende Neuberechnungen nur provisorisch angegeben werden, die Besetzungsdichtigkeit läßt sich mit der des Hauptteiles nicht vergleichen. In Fig. 5 findet man die Projektion einer derartigen Darstellung auf die $\mathrm{Al}_{2} \mathrm{O}_{3}-\mathrm{R}_{2} \mathrm{O}-\mathrm{RO}-$ Ebene. Sie vermittelt gleichzeitig ein einigermaßen perspektivisches Bild, obschon einzelne Kantenrichtungen aufeinanderfallen. AuBerhalb des in dieser Figur gezeichneten Gesamtraumes für Eruptivgesteine liegen verhältnismäbig sehr wenige Analysen magmatischer Gesteine, sofern man von den Erzabsonderungen und Pegmatitbildungen absieht.

Damit sind aber die chemischen GesetzmäBigkeiten noch lange nicht erschöpft. Die verschiedenen Typen der Eruptivgesteine finden sich nicht regellos vermischt vor, auch die Gesteinsassoziation

1) $\mathrm{Fe}_{2} \mathrm{O}_{3}$ mul leider bei derartigen statistiselen Untersuchungen noch $z u$ FeO geschlagen werden, weil die Bestimmungen des Oxydationsgrades yon Eisen in Gesteinen meist unzuverlässig sind. hat ihren besonderen Charakter. Die Gesteine eines Eruptivgebietes weisen bestimmte verwandtschaftliche Züge auf, die oft dem Gesamtgebiet ein eigenes Gepräge verleihen. Anderseits "erkennt man analoge Zusammenhänge in weit entfernten Eruptionsprovinzen wieder; ein deutlicher Hinweis, daB es sich um universelle Abhängigkeiten handelt. Nicht selten gelingt es, die Verwandtschaften chemischer Art diagrammatisch festzulegen und so die Beziehungen zwischen den einzelnen Bestandteilen direkt za erfassen. Der Begriff der Gesteinsassoziation ist zuerst an Hand der Zugehörigkeit gewisser Erzlagerstätten zu.bestimmten Eruptivgesteinen dargelegt worden. Am augenfälligsten tritt er im Ganggefolge tiefenmagmatische Gesteine oder in der Sukzession der Laven ein und desselben Vulkanes zutage. Die ursächlichen Zusammenhänge reichen aber oft viel weiter, und manche Eruptionszyklen und Gesteinsreihen scheinen untex ähnlichen Umständen primär überall gülige Gesetze zu vertreten. So werden granitische und syenitische Gesteine fast stets von pegmatitischen Gängen durchbrochen, deren Intrusion sich direkt an die Erstarrung des Hauptstocks ansehliebt. Zwei Reihen der Gesteinsassoziation kommen in den extremen Gliedern meist getrennt, in den. Mittelgliedern nicht selten gemeinsam vor, die sogenannten pazifischen und atlantischen Sippen (Kalkialkali und Alkalireihen). Zeigt sich in der ersten Reihe eine ziemlich strenge Abhängigkeit des-CaO-Gehaltes der Plagioklase vom Gesamtgehalt dex Basen' z'weif wertiger. Metalle, so fehlt diese in den anderen Ge: steinen, die neben Alkalifeldspäten auch die weniger $\mathrm{SiO}_{2}$-reichen Feldspatstellvertreter (Nephelin, Analcim, Leucit) besitzen. Für die eine Reihe scheinen Gleichgewichtsverschiebunğen nach der Gleichung

$$
2 \mathrm{RSiO}_{3} \rightleftarrows \mathrm{R}_{2} \mathrm{SiO}_{4}+\mathrm{SiO}_{2}
$$

besonders maBgebend zu sein; für die andere Reihe auberdem noch

$$
\mathrm{K}_{2} \mathrm{Al}_{2} \mathrm{O}_{4} \cdot 6 \mathrm{SiO}_{2} \rightleftarrows \mathrm{K}_{2} \mathrm{Al}_{2} \mathrm{O}_{4}+4 \mathrm{SiO}_{2}+2 \mathrm{SiO}_{2}
$$

beziehungsweise

$$
\mathrm{Na}_{2} \mathrm{Al}_{2} \mathrm{O}_{4} \cdot 6 \mathrm{SiO}_{2} \underset{\text { (Fortsetzung folgt.) }}{\longrightarrow} \mathrm{Na}_{2} \mathrm{Al}_{2} \mathrm{O}_{4} \cdot 2 \mathrm{SiO}_{2}+4 \mathrm{SiO}_{2}
$$

\section{Die Entstehung der Kurzsichtigkeit.}

Ton Augenarzt Prof. Dr. G. Levinsonn, Berlin.

Die hohe Bedeutung einer Lösung des Problems von der Entstehung der Kurzsichtigkeit liegt hauptsächlich darin, daß mit einer Aufdeckung der Entstehungsursache gleichzeitig. auch die richtige Waffe gegen die ungeheure Verbreitung der Kurzsichtigkeit und ihre zahlreichen Schädigungen gefunden ist. Wenn trotz der auberordentlich zahlreichen Bemühungen auf diesem Gebiete die erzielten Erfolge nur recht minimal genamnt werden können, so mub der wesentliche Grund für diese Erscheinung auf die 
zurzeit, völlig ungenügende Erkenntnis des genannten Problems zurückgeführt werden.

\section{Die Kursichtigheit eine Folge der Naharbeit.}

Da es seit langem bekamnt und durch zahlreiche Statistiken immer von neuem bewiesen ist, daß die überwiegende Mehrzahl aller Fälle von Kurzsichtigkeit in der Schule entsteht, und daB die Kurzsichtigkeit um so mehr gefördert wird, je größer die Anforderungen sind, welche an den Sehuler gestellt werden, so hat man in erster Linie die Naharbeit für die Entstehung der Kurzsichtigkeit geltend gemacht. Die meisten und bekanntesten Myopietheorien basieren infolgedessen darauf, daß 3 es die bei der Naharbeit wirksamen Faktoren, die Konvergenz und Akkomodation des Auges sind, deren verstärkter Tätigkeit die Entstehung der Kurzsichtigkeit zuzuschreiben ist. Naturlich handelt es sich hier nur um die Fälle ron Achsenmyopie, die Form der Kurzsichtigkeit, welche durch Verlängerung des Auges zustande kommt, nicht um die seltenen falle von Kurzsichtigkeit infolge vermehrter Brechung der Augenmedien, insbesondere der Linse, Fälle, die vorzugsweise im späteren Lebensalter zur Beobachtung gelangen. Das Zustandekommen der Dehnung durch Konvergenz oder Akkomodation führte man auf die vermehrte Tätigkeit der äuBeren resp. des inneren Augenmuskels zurück, durch welche das Auge unter einen erhöhten Druck gebracht und wegen seiner im jugendlichen Zustande vorhandenen Nachgiebigkeit allmählich gedehnt werden soll. $\mathrm{Da}$ die Verlängerung des Auges im sagittalen Durchmesser um $1 \mathrm{~mm}$ schon eine Kurzsichtigkeit von $3 \mathrm{D}$. bedingt, so genügt ja schon ein geringer Grad von Dehnung, um auf diese Weise leicht Kurzsichtigkeit zu erzeugen resp. diese, wo sie schon besteht, allmählich zu erhöhen.

\section{Unrichtigkeit der bisherigen Myopietheorien.}

Alle diese Muskeltheorien sind auf zwei falschen Voraussetzungen aufgebaut. Einmal ist mit Sicherheit auszuschließen, daß die bei der Kurzsichtigkeit auftretenden sehr charakteristischen anatomischen Veränderungen durch die Drucksteigerung im Auge entstanden sind. Wir sind über die Folgen dieser Drucksteigerung sowohl im jugendlichen wie im Auge des Erwachsenen sehr genau orientiert. Wir wissen sowohl aus sehr zahlreichen klinischen Beobachtungen, als auch exakten anatomischen Untersuchungen, daf die bei der Kurzsichtigkeit auftretenden Veränderungen im Auge von denjenigen, die bei. Druckvermehrung des Auges gefunden werden, völlig verschieden sind, daß beide nicht das geringste miteinander gemein haben. Dann aber haben die Untersuchungen von $H e \beta$ and $H e i n e^{1}$ für die Akkomodation, sowie die Untersuchungen des Ver-

1) Hep und Heine, v. Gräfes Archiv f. Ophthalm. Bd. 46,2 . fassers ${ }^{1}$ ) für die bei der Naharbeit in Frage kommenden Bewegungen der Augenmuskeln einwandfrei bewiesen, daf eine Druckvermehrung hierbei überhaupt nicht existiert, oder daB diese so minimal ist, daß sie nie und nimmer eine anatomische Veränderung hervorrufen kann. Mit diesem Nachweis sind alle Muskeltheorien erledigt. Das gilt auch für die sehr beliebte Stillingsehe Theorie, nach welcher die Dehnung des Auges ron einem bestimmten Muskel abhängen (Muscul. obliq. super.) und weniger die Folge einer direkton Muskelwirkung sein, als durch den Widerstand hervorgerufen werden soll, welchen das Auge wälnrend des Wachstums an diesem Muskel findet. Abgesehen daron, dal dieso Theorie die verstärkte Tätigkeit eines Muskels während der Naharbeit annimmt, der in Wirklichkeit lı̈̈chstwahrscheinlich sehr wenig in Aktion tritt, und dal sie gleichtalls eine Drucksteigerung voraussetzt, dit. bestimmt nicht vorhanden ist, daB sie ferner den anatomischen Veränderumgen bei der Kurzsichtigkeit ganz und gar nieht Rechnung trägt, wird hier ein neuer Faktor in Rechnung gesetzt, ler bei der Entstehung der Kurzsichtigkait zweifellos so gut wie keine Rolle spielt. Das ist der Faktor des Wachstums. Wir wissen, daB das Wachstum des kindlichen Auges mit dem 4. Lebensjahre ziemlich abgeschlossen ist. Voul dieson bis zum 14. Jahre, also in der Zeit, in der die Kurzsichtigkeit rorzugsweise entsteht, wächst las Auge nur sehr minimal, etwa $1 \mathrm{~mm}$ in allen Durchmessern. Wenn aber ein Wachstum übcrbaupt nicht existiert, kann das Ange auch nicht durch dieses in eine falsche Richtung gedränot werden.

Unberücksichtigt geblicben ist die Tatsache. daß das Wachstum des kindlichen Auges nur sehr geringfügig ist, auch von den Anhängern derjenigen Theorie, die die Kurzsichtigkeit auf einen angeborenen Entwicklungsmangel der deu Selnerveneintritt umgebenden Gewebe (Jäger, Schnabel, Elschnig) zurückführen. Da das Auge während der Entstehung der Kurzsichtigkeit in seinem Wachstum fast vollständig stillsteht, kann naturgemäß die angeborene Anlage auch nicht durch Wachstum vergrößert werden. Dazu kommt, daB diese Theorie das für die Entstehung der Kurzsichtigkeit einzig bewiesene Faktum, die Naharbeit, völlig unberüclssichtigt läßt, und daß bei Kindern sowohl anatomisch, als auch klinisch (Untersuchungen des Verfassers bei Kindern im 1. Lebensjahr) die für Myopie charakteristischen Veränderungen fast nie beobachtet werden.

Also um es noch einmal kurz zusammenzufassen: alle diese Theorien gehen von nachweislich falschen Voraussetzungen aus; aber selbst wenn diese Voraussetzungen richtig wären, so würden die Theorien im Widerspruch stehen mit den charakteristischen anatomischen Verände-

1) G. Levinsonn, v. Gräfes Archiv f. Ophthahn. Bd. $\gamma 6,1$, und Archiv i. Augenheilk. Bd. 73, H. $2 \%$. 
rungen, die bei der Kurzsichtigkeit angetroffen werden.

\section{Die Kurzsichtigkeit eine Folge dev Rumpf-und Ropfbeugung.}

Gegenübex dem völligen Versagen der Myopietheorien ist es dem Verfasser gelungen, eine Theorie über die Entstehung der Kurzsichtigkeit aufzustellen, welche dem bisher in der Myopiefrage angesammelten Tatsachenmaterial in hohem Grade gerecht wird und die außerdem durch einschlägige Versuche als einwandfrei erhärtet ist ${ }^{1}$. Die Theorie geht davon aus, daß bei der Naharbeit gleichzeitig mehr oder weniger eine Beugung des Rumpfes und Kopfes stattfindet, und dab demnach das Auge hierbei nach vorn fallt und der Schwerkraft der Erde unterliegt. Da das Auge im jugendlichen Zustand einen ziemlich beträchtlichen Grad von Dehnungsföhigkeit besitzt. so muB naturgemäB in diesem eine Dehnung stattfinden, etwa in dem Sinne, wie ein mit Wasser gefüllter an einem Faden aufrehängter Gummiball gedehnt wird. Je länger und je intensiver die Rumpf- und Kopfbengung austält, um so stärker muß die Dehnung, um so wriber die Kurzsichtigkeit sein.

Beweise für die Rivltiglseit der obigen Theorie.

Die Richtigkeit dieser Theorie wurde znnächst dadurch bewiesen, dab nach Befestigung einer luftdichten, dem Auge angepaBten Guttaperchakapsel, die mit einem Gummischlauch, Mareyscher Kapsel und Kymographion in Verbindung stand, die starke Verschiebung der Luftsäule bei jeder Rumpf- and Kopfbeugung in Kurven graphisch fixiert werden konnte. Diese Versuche decken sich mit den Beobachtungen von Birch-Hirschfold'), der das Vorrucken des Anges béi der Kopfbeugung in exakter. Weise photographisch festgelegt hat. Das Auge sank nach diesem Autor bei der Kopfbeugung um $90^{\circ} 1,7 \mathrm{~mm}$ nach vorn. Da dex Sehnerr in der Orbita einen leicht geschlängelten Verlauf besitzt, so konnte der Einwand erhobew werden, der auch in der Tat erhoben worden ist, daB die Schlängelung desselben eine durch die Kopfbeugung bedingte Zerrung unmöglich macht. Intersuchungen von Weip haben aber gezeigt, dab durch die während der Naharbeit stattfindende Konvergenz der Sehnerv mehr oder weniger gestreckt wird, so daB in riesem Zustand das zerrende Moment der Schwerkraft roll und ganz auf ihn einwirken kamn. Dazu kommt, daß die den Sehnerv umgebenden Widerstände, vor allem das prall gefüllte Fettpolster, daß ferner die dem Sehnerv innewohnende Starre und die Befesti-

1) G. Lewinsohr, Das wesentliche Moment bei der Entstehung d. Kurzsichtigkeit. Bericht uber die 35. Versamml. d. ophthalm. Gesellseh. Heidelberg 1908, and Die Entstehung der Kurzsichtigkeit. Ferlag S. Karger, Berlin 1912.

2) Birch-Ifirschfeld. Die Krankheiten dar Orbita. Gräfe-Sämisch, Handb. d. gesamten Allgenheilkunde. 2. Aufl. gung desselben nicht weit von seinem Eintritt in das Ange durch die ZentralgefäBe, eine vollständige Streckung desselben bei der Beugung verhindern und dadurch die schädigende Einwirkung der Schwerkraft auf das Auge in ganzem Umfange zur Geltung kommen lassen.

Diese Zerrung muß nun Veränderungen herrorrufen, welche sich mit denjenigen, die bei der Kurzsichtigkeit angetroffen werden, vollkommen decken. Die für Myopie charakteristischen Veränderungen bestehen bekanntlich in sichelformigen atrophischen Partien der Aderhant, die ihren Anfang von dem Schläfen-, ein wenig nach unten gerichteten Rande des Sehnerveneintritts nehmen und sich entweder nach der Schläfenseite immer mehr entwickeh oder mit der Zeit den ganzen Sehnerven umkreisen. Dazu kommen Dehnungen und Zerrungen der Aderhaut im Mittelpunkt des Auges, Verschiebungen der Netz- und Aderhaut am Nasenrande des Selnerveneintritts, Schlängelung der Sehnervenfasern und anderes mehr. Weun man berücksichtigt, dab der Sehnerv ron der Nase wach der Schläfenseite zieht, so muB die Schwerkraft das Auge am Sehnerveneintritt aus rein physikalischen Grinden bei der Koptbeugung einmal nach dex Nasenseite zu verschieben, andererseits rom Sehnerven loszutrennen die Tendenz haben. Das aber sind die beiden Faktoren, welche das Zustandekommen der oben angeführten Veränderungen äuBerst plausibel machen und es sehr gut verstehen lassen, warum gerade die ensten Veränderungen am Schläfen-, meist ein wenig nach unten gerichteten Rande des Sehnerveneintritts auftreten. Natürlich werden angeborene Abweichungen im anatomischen Verhalten des Selnerveneintritts auf die Art der durch die Schwerkraft bedingten Veränderungen nicht ohne Ginflub bleiben, im großen ganzen aber werden die bekannten und sowohl in ihrem Beginn wie in der späteren Entwicklung der Kurzsichtigkeit charakteristischen Veränderungen die Regel bleiben. Die sehr plausible Deutung der bei Kurzsichtigkeit auftretenden anatomischen Veränderungen durch Beugung des Kopfes muB uim so mehr betont werden. als nach den bisherigen Theorien gerade diese Veränderungen eine sehr gekïnstelte und zum Teil widerspruchsvolle Erklärung gefunden hatten.

\section{Naharbeiter ohne Kopfbeugung uerden nicht kurz- sichtig.}

Anch noch aus einem anderen Grunde ist die ron dem Verfasser anfgestellte Theorie geeignet, aufklärend $z u$ wirken. Es war immer aufgefalJen, daß unter den Berufsklassen, welche sich mit intensivster Naharbeit heschäftigten, bei den Uhrmachern, Juwelieren and Goldarbeitern, ferner bei den Feinstickerimnen der Prozentsatz an Kurzsichtigkeit ein relativ geringer blieb, Man hat diese sehr auffallende Tatsache durch künstliche Hypothesen zu erklären versucht. Die einen führten den rerringerten Prozentsatz darauf zu- 
rïck, daß die Vertreter dieser Berufe gewöhnlich nur mit dem einen Auge, also verringerter Konvargenz arbeiten, die anderen darauf, dab in diesen Berufen die Augen während der Arbeit keine kleinen zuckenden Bewegungen wie beim Lesen ausführen. Diesen ganz willkürlichen Vermutungen gegenüber mag nochmals auf die Untersuchungen des Verfassers hingewiesen sein, welche gezeigt haben, daß sowohl bei der. Konvergenz als auch den kleinen Bewegungen des Auges entweder gar keine oder so gut wie keine Drucksteigerung und daher auch keine Dehnung stattfindet, ferner, dab die bei dex Kurzsichtigkeit gefundenen Veränderungen dieser Theorie aufs strikteste widersprechen. Die Erklärung für den geringen Prozentsatz an Kurzsichtigkeit bei den genannten Berufsklassen ist eine sehr einfache und deckt sich vollständig mit der von dem Verfasser aufgestellten Theorie. Die Uhrmacher, Juweliere und Goldarbeiter arbeiten nämlich auf niedrigen SchemeIn an hochgestellten Arbeitstischen, die Feinstickexinnen haben ihre Arbeiten in großen Ralmen ausgespannt, welche dem Auge gerade gegenubberstehen, oder die in einem kleinen Rahmen eingeschlossene Arbeit wird durch ein Schraubgewinde dem Auge möglichst genähert. In allen diesen Fällen wird daher die Arbeit mit stark angenähertem Auge, aber in einer relativ geraden Haltung verrichtet, und das schädigende Moment der Rumpf- und Kopfbeugung spielt bei diesen Arbeitern nur eine geringe Rolle.

\section{Experimentelle Erzeugung der Kurzsichtigkeit.}

Die angeführten Beweise für die Richtigkeit obiger Theorie waren bisher nur theoretischer Natur. Wenn diese Theorie richtig ist, so muBte es auch gelingen, auf der Grundlage derselben Kurzisichtigkeit experimentell zu erzeugen. Der Verfasser hat daher Tierversuche angestellt, z11nächst an jungen Kaninchen, Hunden und Katzen, die täglich für mehrere Stunden des Tages in eine Stellung gebracht wurden, bei der der Kopf nach abwärts gerichtet war. Die Tiere, die diese Prozedur sehr gut vertrugen, wunden nach vorheriger sorgfaltiger Refraktionsbestimmung unter Atropin, welches die Akkomodation lähmte, in verschiedenen Zwischenräumen untersucht. Es gelang, auf diese Weise 12 Tiere $z u$ exhalten, die nach längerer resp. kürzerer Zeit ( 8 bis $90{ }^{\text {T}}$ Tagen) eine allmählich zunehmende Kurzsichtigkeit von 1 bis 4,5 D. aufwiesen. Ophthalmoskopische Veränderungen wurden allerdings an diesen Tieren nicht beobachtet. Diese Versuche sind ron Posseh ${ }^{1}$ ) nach geprüft und in vollem Umfang bestätigt worden.

Aber diese Versuche befriedigten den Verfasser nicht ganz. Finmal, weil es sich um Augen landelte, deren anatomischer Bau dem mensch-

1) R. Possek, Die Ursachen, Veruutung und Behandlung der. Sehstorungen bei Sehulkindern. 17. intexaat. Fongres London, Sektion XVIII. lichen gegeuüber nicht unwesentliche Unterschiede aufweist, dann aber, weil die Vensuchsanordnung bei diesem Tienmaterial eine ziemlich grobe sein mubte. Die Vensuche wurden daher wiederholt und zwar an jungen Affen, deren Augen mit denjenigen des Menschen eine sehr grobe Ahnlichkeit besitzen ${ }^{1}$ ). Die Affen wurden in kleine der Gröbe der Ticre entsprechende Kästchen eingeschlossen, so daß der Kopf oben herausragte und. frei beweglich blieb, aber durch Verlängerung der Rückenwand der Kästchen vexhindert wurde, sich über die Wirbelsäule nach rückwärts zu bewegen. Die Kästchen wurden dann leicht schräg, etwa in einem. Winkel von $10^{\circ}$ zur Tischoberfläche aufgestellt und der Affe infolgedessen gezwungen, die Augen senkrecht nach abwärts zu richten; die Entferuung $z w i s c h e n$ dem Knotenpunkt der Augen und der Tischplatte, auf der einige Rüben lagen, betrug $14 \mathrm{~cm}$. Die Augen befanden sich hier in einer Stellung, die mit derjenigen des Kindes

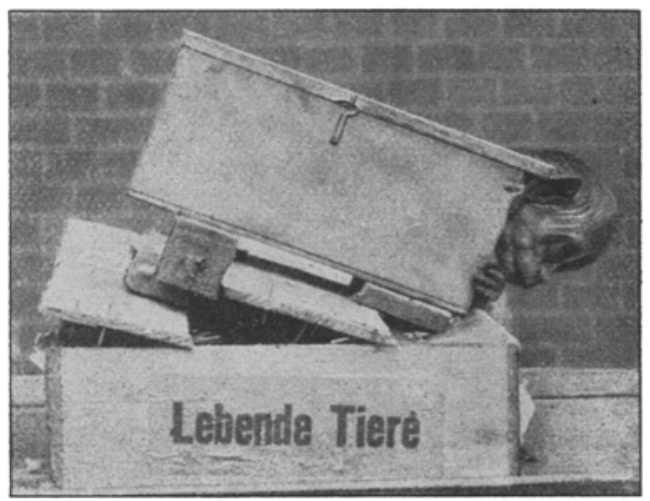

Versuchsanordnung zur absichtlichen Erzeugung von Kurzsichtigkeit an Affen: der Affe wird veranlaBt, die Augen andauernd senkrecht nach unten gu richten. Das Auge fällt hierbei unter der Einwirkung der Schrerkitit nach vorn.

beim Schreiben und Lesen sehr große Ahnlichkeit besitzt. Zum besseren Verständnis der Versuchsanordnung ist dieselbe hier im photographischen Bilde wiedergegeben. Die täglich für einige Stunden ausgeführte, sehr harmlose Prozedur ertrugen die Tiere ohne die geringste Gesundheitsschädigung. Von sechs Affen, unter denen sich zwei Kontrolltiere befanden, gingen abex vier Tiere an Tuberkulose schon nach kurzer Zeit ein, die beiden anderen wurden 9 und 12 Monate am Leben erhalten. Bei dem einen normalsiehtigen Affen betrug die Refraktion des Auges am Ende der Tntersuchung $-9 \mathrm{D}$. und -7 D., bei dem zweiten Affen, der schon bei Beginn der Untersnchung eine geringe Kurzsichtigkeit aufwies,

1) G. Levinsohn, Die Entstelung der Kurzsichtigkeit, mit Demonstration kurzsichtig gemachter Affen. Bericht uber die 39. Tersamml. d. ophthalm. Gesellschaft, Heidelberg 1913. 
(-3 D.), steigerte sich diese im Verlauf von 12 Monaten auf $-14 \mathrm{D}$. und $-15 \mathrm{D}$. Die $\mathrm{Zu}$ yahme der Refraktion erfolgte langsam und allmählich, etwa alle 8 bis 14 Tage um $1 / 2$ D., in der letzten Zeit wurde häufig ein Stillstand in der Zumahme beobachtet. Gleichzeitig mit dem hohen Grade der Kurzsichtigkeit traten nun Veränderungen am Rande des Sehnerven auf. die sich langsam verstärkten, aber erst nach Monaten deutlich in Erscheinung traten. Es handelte sich um eine dextliche Konusbildung am Schläfenund eine Supertraktionsbildung am nasalen liande, Veränderungen, die für Kurzsichtigkeit geradezu typiseh sind.

AuBerordentlich charakteristisch und von ganz besonderer Bedentung war dann der histologische Befund dieser Augen ${ }^{1}$ ). Dieser war ausgezeichnet durch alle. diejenigen Merkmale, die bei der Tiurzsichtigkeit am mensehlichen Auge so oft zur Beobachtung gekommen sind, nur dab sie beim Iffen in einer Reinheit und Ubersichtlichkeit zutage traten, wie sie beim Menschen infolge dex sekundären. Verändernngen fast nie angetroffen wrerden.

\section{Wichtigkeit dor affenversuche.}

Den Versuchen am Affenauge muß eine ganz besondere Bedeutung zuerkannt werden,

1. weil die Kurzsichtigkeit bei Affen, abgesehen ron vereinzelten Fällen geringen Grades im allgemeinen unbekannt ist, jedenfalis hochgradige Turzsichtigkeit wie bei den Versuchstieren niemals beobachtet worden ist,

2. weil die hier nachgewiesenen histologischen Veränderungen gleichfalls noch niemals beim Affen festgestellt sind,

3. weil die Entwicklung der Myopie, das Auftreten der ophthalmoskopischen Abweichungen und die Ausbildung der histologisehen Veränderungen einer sehr harmlosen Versuchsanordnung. nämlich der voribergehenden Horizontalstellung. ler Tiere ihre Entstehung verdanken.

Durch die Versuche der Affex ist der exakte Beweis erbracht, daB in der Tat nur in der Beuwung des Rumpfes und Kopfes und der dadurch bedingten Einwirkung der Schwerkraft auf das Ange dasjenige schädigende Moment der - Naharbeit gesucht werden muk, das die Dehinung des Auges, d. h, Kurzsichtigkeit, zur Folge hat.

\section{Disposition für die Entstehung der Kurz- sichtigheit.}

Es bleibt nun noch übrig, auf diejenigen Faktoren, welche neben der Beugung des Rumpfes wid Kopfes auf die Entstehung der Kurzsichtigkeit einen Einfluß besitzen, mit kurzen Worten cinzugehen. Da kann es zunächst keinem Zweifel unterliegen, daß die Kurzsichtigkeit sich vorzugs-

i) a. Levineolin, Uber den histolog. Befund kurz sichtig gemachter Affenaugen und die Entstehung der Kurzsichtigkeit. v. Gräfes Archiv : Oplthalmologie Bd. $88,3$. Heft, S. 459, 1914 . weise in denjenigen Augen entwickeln wird, die für die Dehnung eine besondere Disposition besitzen. Das Moment der Disposition spielt natürlich bei der Kurzsichtigkeit die gleiche Rolle, wié bei allen anderen Krankheiten resp. Entwicklungsstörungen. Bezüglich der ererbten Anlage wird man aber nicht umhin können $\mathrm{zu}$ berücksichtigen, daB über $50 \%$ allex Gebildeten durch die Schnle kurzsichtig werden, und daB' es daher gar nicht iberraschen kann, wenn so auberordentlich häufig wenigstens der eine der Eltern kurzsichtig gefunden wird. Dab abgesehen von den Eltern in den weiteren Graden der Verwandtschaft fast immer Kurgsichtige angetroffen wexden, ist unter diesen. Umständen geradezu selbistverständlich. Von viel größerer Bedeutung als die angeborene Veranlagung scheint die erworbene Disposition zu sein. Es hat sich gezeigt, daB die Kurzsichtigkeit vorzugsweise bei schwächlichen Individuen aufzutreten pflegt, namentlich ist auch die Beobachtung gemacht worden, dab Myopie sich im Gefolge erschöpfender Trankheiten, wie Scharlach, Masem, Diphtherie, einzustellen pflegt. Der Grund für diese Erscheinung liegt darin, daß durch diese Krankheiten alle Gewebe, demnach auch die Eederhaut des Auges, in ihrer Widerstandsfähigkeit geschädigt werden, und das letztere dem dehnenden Zuge der Schwerkraft um so leichter Folge leistet.

Eine besondere Disposition für die Ausbildung der hoheren Grade von Kurzsichtigkeit kommt den Fällen mit angeborener Myopie zu. Diese Fälle sind allerdings nur selten. Von früheren Untersuchern ist das seltene Vorkommen angeborener. Kurzsichtigkeit fast durchweg betont worden. Untex 50 Kindern im ersten Lebensjahr hat der Verfasser nach Akkomodationslähmung unter Atropinmydriasis nur bei einem Kinde eine Kurzichtigkeit von 4 und 4,5 D. festgestellt, bei der großen Mehrzahl aller Kinder war der Bau des Auges mehr oder weniger übersichtig. 'Die hochgradige Kurzsichtigkeit dürfte daher nur selten eine angeborene sein und sich in der Regel auf der Basis der Normal- resp. Übersichtigkeit entwickeln. Ein prinzipieller Unterschied zwischen einem weniger und hochgradig kurzsichtigen Auge besteht nicht. Die Trennung in zwei ganz verschiedene Formen (Sehul- und angeborene Kurnzichtigkeit) ist rẹn willkürlich und nur ron klinischen Gesichtspunkten aus gemacht worden, da sichere Grenzen zwischen beiden Formen fehlen. Wir können während der Fntwicklung fast niemals mit Sicherheit feststellen. welche Fol'm vorliegt, und ob nicht die letztere in die erstere übergehèn wird, ror allem aber ist das anatomische Substrat in beiden Formen durchaus das gleiche und nur - auch nicht einmal immer - dem Grade nach verschieden. Inwieweit bei dem Auftreten hochgradiger Kurzsichtigkeit, da wo die Naharbeit keine Rolle gespielt hat, also insbesondere bei Landarbeitern, neben der Veranlagung - angeborener oder er- 
worbener Disposition - das Moment der Rumpfund Kopfbeugung wirksam gewesen ist, mag hier unerörtert bleiben, da klinische Untersuchungen nach diesex Richtung nicht volliegen.

\section{Wirksame Bekämpfung der Kurzsichtigkeit.}

Die Auffassung, daB bei der Entstehung der Kurzsichtigkeit als selädigendes Moment in erster Linie die Rumpf- und Kopfbeugung in Frage kommt, kann auf Grund der angeführten Tatsachen nicht bezweifelt werden. Eine wirksame Bekämpfung wird daher nur Aussicht auf Erfolg haben, wenn sie vorzugsweise auf Ausmerzung dieser Schädigung beim wachsenden Individuum gerichtet ist. In zweiter Linie wird eine Herabsetzung der Disposition, welche das Auftreten der Kurzsichtigkeit begünstigt, angestrebt werden müssen. Aufgabe der Hygiene, insbesondere auf dem Gebiete der Schule, wird es sein, durch Aufklärung der Bevölkerung und geeignete Maßnahmen der außerordentlichen Verbreitung der Kurzsichtigkeit mit allen ihren Schädigungen wirksam zu begegnen.

\section{Besprechungen.}

Planck, M., Eight lectures on theoretieal physics, delivered at Columbia University in 1909. Trans. lated by A. P. Wills. New York, Columbia University, 1915, $130 \mathrm{~s}$.

Dif acht Vorlesungen, die Max Planck im Frühjahr 1909 als foreign lecturer an der Columbia-Universität gehalten hat, sind in deutsclier Sprache sehon im Jahre 1910, also drei Jahre vor der Gründung dieser Zeitschrift, im Verlag von S. Hirzel (Leipzig) erschienen. Da infolgedessen die Bedentung des Werkes an dieser Stelle noch nicht gewuirdigt worden ist, so sei es jetzt, bei Gelegenheit des Erscheinens der englischen Ubersetzing; gestattet, die Besprechung nachzuholen.

Planch: hat in diesem liöchst lesenswerten Buche den damạligen Stand des Systems dex theoretischen Physik meisterhaft gezeichnet. In verhältnismüBig knapper Form, wie sie durch die Natur der Vorlesung geboten war, findet der Leser hier wichtige Abschnitte ans der Thermodynamik, der kinetischen Gastheorie, der Wärmestrahlung und der Relativitätstheorie behandelt. Uberall ist der Kern der Probleme in schöner und lichtvoller Weise herausgeschält, überall die Originalität der. Darstellung gewahrt; denn auf allen diesen Gebieten sind Plancks eigene Forschumgen bahnbrechend und fördernd gewesen. Manche Entwickelungen, besonders im Gebiet der Quantenlehre, haben in den letzten sieben Jahren Veränderungen erfaliren, zum Teil hat sie Planck selbst modifiziert. Auch ist durch die Schaffung der Einsteinschen Gravitationstheorie die Relativitatstheorie bedeutend verallgemeinert und erweitert worden. Trotzdem ist, dem Wunsche des Verfasser's gernati, bei der englischen Utbersetzung von einer Veränderung des Originals abgesehen worden. Und dies mit vollem Recht! Denn nur so kann man in Gebieten, wo die Ideen in starkem Flusse sind, den historischen Entwicklungsgang der fraglichen Theorien verfolgen und durch Vergleich der damaligen mit den hentigen Anschaungen die Fortschritte einschätzen.
Den Inhalt des Buches wollen wir kurz skizzieren. Die erste Vorlesung wirft die Frage auf, in welchex Weise man am sachgemäbesten das System der theo. retischen Physik gliedern könne. Planck entscheidet sich füx die Einteilung allex physikalischen Prozesse in reversible und irreversible. Der Begriff and die Existenz irreversibler Vorgänge in der Natur sind engt verknüpft mit dem zweiten Hauptsatz der Thermodynamik, der durch Einführung des Entropiebegriffes nach clansins formuliert wird. Eine rein thermodynamische Anwendung des zweiten Hauptaatzes bringt die zweite Vorlesung. Hier werden durch Benutzing eines an Gibbs anknitpfenden thermodynanischen $\mathrm{Po}^{-}$ tentials die Gleichgewichtssustände in verdünten. Lösungen nach einer einheitlichen Metbode behandelt. und so $z$. B. die van't Ifoffichen Gesetze der Siedepunktserhohung der Gefrierpunkt - und Dampfdruekenniedrigung und des asmotischen 1)ruckes gewonnen.

In der folgenden. Vorlenung wircl der Entropiebegriff durch Einführung der atomistisehen Theori* der Materie vertieft und die Boltzmannsche Beziehmug zwischen Eutropie und Znstandswahrischeinlichkeit he?gestellt. Auf Grund diaser fundamentalen Beziehung werden in der vierten Vorlesung die Gesetze abgeleitot. die das Verhalten der idealen Gase in cilejohgewichtwzustand beherrschen, das Maxwellsche Vertellumgsgestot der Molekulgeschwindigkeiten und die Gesetze ron Boyle, Gay-Lussac und Avogadro. Die fünfte und sechste Vorlesung enthalten die Plancksche Theorie dix Wärmestrahlung. Nach einer Ubersicht iuber die Grundbegriffe dieser Lehre wird zuerst der rein elektrodynamisehe Teil bebandelt, nämlich das bekannte Problem des Resonators: im Strahlungsfelde. dessem Durchführung in der Beziehung zwisclien der spektralen Strahlungsintensitat des Feldes und der mittleren hesonatorenergie gipfelt. Der zweite Teil der Theorie ist statistiseher Natur. In inm wird die Grundidee der Qrautenvorstellung entwickelt, in der Form, daB den Flementargebieten gleicher Wahrscheinlichkeit in der Zustandsebene des Resonators endliche Gröbe zukommt. Es ist dies wesentlich diesclbe Formulierung der Quantenhypothese, die Plunck in seinen jüngsten Arbeiten uber die Struktur des Phasenraumes erweitert und ausgebut lat. Aus der Quantenforderung folgert er die ganzzahligen diskreten Energiewerte des Resonators. Unter der Voraussetzung dieser quantenhaften Teilung der Resonatorenergie wird die Wahrscheinlichkeit für einen bestimmten Zustand des Resonators und daraus mit Hilfe der Boltzmnnschen Beziehmo die Entropie des Resonators als Funktion seiner mittleren Energie berechnet. Durch Einführung der Temperatur bei Anwendung des zweiten Hauptsatzes und Vèrknüpfung mit dem Resultat des elektrodynamischen Teiles folgt schließlieh die spektrale Strahlungsintensititt als universelle Funktion von Schwingungszahl und Temperatur, d. h. das Strahlungsgesetz des schwarzen Körpers.

Die beiden letzten Vorlesungen sind der allgemainen Dynamik gewidmet. Hier wird das Hamiltonsche Prinzip der kleinsten. Wirkung als beherrschende Macht aufgestellt und seine anBerordentliehe Fruehtbarkeit an Beispielen aus der Mechanik, der Thermodynamik und der Elektrodynamik erläutert.

Den SchluB bildet ein kurger AbriB des LorentzEinsteinschen Relativitätsprinzips, seiner Entstehungsgeschichte, seiner mathematischen Formulierung und seiner weittragenden Folgen in den versohiedenen Gebieten der Physik.
F. Reiohe, Berlin. 